\title{
Association between type 2 diabetes genetic susceptibility loci and visceral and subcutaneous fat area as determined by computed tomography
}

\author{
Kikuko Hotta ${ }^{1}$, Aya Kitamoto ${ }^{1}$, Takuya Kitamoto ${ }^{1}$, Seiho Mizusawa ${ }^{2}$, Hajime Teranishi ${ }^{2}$, Rina So ${ }^{3}$, \\ Tomoaki Matsuo ${ }^{3}$, Yoshio Nakata ${ }^{3}$, Hideyuki Hyogo ${ }^{4}$, Hidenori Ochi ${ }^{4}$, Takahiro Nakamura ${ }^{5}$, Seika Kamohara ${ }^{6}$, \\ Nobuyuki Miyatake ${ }^{7}$, Kazuaki Kotani ${ }^{8}$, Ryoya Komatsu' ${ }^{9}$, Naoto Itoh ${ }^{10}$, Ikuo Mineo ${ }^{11}$, Jun Wada ${ }^{12}$, \\ Masato Yoneda ${ }^{13}$, Atsushi Nakajima ${ }^{13}$, Tohru Funahashi ${ }^{14}$, Shigeru Miyazaki ${ }^{15}$, Katsuto Tokunaga ${ }^{16}$, \\ Hiroaki Masuzaki ${ }^{17}$, Takato Ueno ${ }^{18}$, Kazuaki Chayama ${ }^{4}$, Kazuyuki Hamaguchi ${ }^{19}$, Kentaro Yamada ${ }^{20}$, \\ Toshiaki Hanafusa $^{21}$, Shinichi Oikawa ${ }^{22}$, Hironobu Yoshimatsu ${ }^{23}$, Toshiie Sakata ${ }^{23}$, Kiyoji Tanaka ${ }^{3}$, \\ Yuji Matsuzawa ${ }^{8}$, Kazuwa Nakao ${ }^{1,24}$ and Akihiro Sekine ${ }^{1,2}$
}

Visceral fat accumulation has an important role in the development of several metabolic disorders, such as type 2 diabetes, dyslipidemia and hypertension. New genetic loci that contribute to the development of type 2 diabetes have been identified by genome-wide association studies. To examine the association of type 2 diabetes susceptibility loci and visceral fat accumulation, we genotyped 1279 Japanese subjects (556 men and 723 women), who underwent computed tomography for measurements of visceral fat area (VFA) and subcutaneous fat area (SFA) for the following single-nucleotide polymorphisms (SNPs): NOTCH2 rs10923931, THADA rs7578597, PPARG rs1801282, ADAMTS9 rs4607103, IGF2BP2 rs1470579, VEGFA rs9472138, JAZF1 rs864745, CDKN2A/CDKN2B rs564398 and rs10811661, HHEX rs1111875 and rs5015480, TCF7L2 rs7901695, KCNQ1 rs2237892, KCNJ11 rs5215 and rs5219, EXT2 rs1113132, rs11037909, and rs3740878, MTNR1B rs10830963, DCD rs1153188, TSPAN8/LGR5 rs7961581, and FTO rs8050136 and rs9939609. None of the above SNPs were significantly associated with VFA. The $F T O$ rs8050136 and rs9939609 risk alleles exhibited significant associations with body mass index (BMI; $P=0.00088$ and $P=0.0010$, respectively) and SFA $(P=0.00013$ and $P=0.00017$, respectively). No other SNPs were significantly associated with BMI or SFA. Our results suggest that two SNPs in the FTO gene are associated with subcutaneous fat accumulation. The contributions of other SNPs are inconclusive because of a limitation of the sample power.

Journal of Human Genetics (2012) 57, 305-310; doi:10.1038/jhg.2012.21; published online 1 March 2012

Keywords: computed tomography; FTO; Japanese subjects; subcutaneous fat area; type 2 diabetes; visceral fat area

\section{INTRODUCTION}

Metabolic syndrome is defined by four conditions: visceral fat obesity, impaired glucose tolerance, dyslipidemia and hypertension. ${ }^{1}$ Various adipocytokines secreted from adipocytes have been identified and shown to cause dyslipidemia, hypertension and insulin resistance. ${ }^{2,3}$ Previous studies on adipocytokines indicate that visceral fat obesity has a central role in the development of metabolic syndrome. The determination of visceral fat mass is performed in terms of waist

${ }^{1}$ EBM Research Center, Kyoto University Graduate School of Medicine, Kyoto, Japan; ${ }^{2}$ Center for Genomic Medicine, Unit of Genome Informatics, Kyoto University Graduate School of Medicine, Kyoto, Japan; ${ }^{3}$ Graduate School of Comprehensive Human Sciences, University of Tsukuba, Tsukuba, Japan; ${ }^{4}$ Department of Medicine and Molecular Science, Division of Frontier Medical Science, Programs for Biomedical Research, Graduate School of Biomedical Sciences, Hiroshima University, Hiroshima, Japan; ${ }^{5}$ Laboratory for Mathematics, National Defense Medical College, Tokorozawa, Japan; ${ }^{6}$ Health Science University, Yamanashi, Japan; ${ }^{7}$ Department of Hygiene, Faculty of Medicine, Kagawa University, Kagawa, Japan; ${ }^{8}$ Department of Metabolic Medicine, Graduate School of Medicine, Osaka University, Osaka, Japan; ${ }^{9}$ Rinku General Medical Center, Osaka, Japan; ${ }^{10}$ Toyonaka Municipal Hospital, Osaka, Japan; ${ }^{11}$ Otemae Hospital, Osaka, Japan; ${ }^{12}$ Department of Medicine and Clinical Science, Okayama University Graduate School of Medicine, Dentistry and Pharmaceutical Sciences, Okayama, Japan; ${ }^{13}$ Division of Gastroenterology, Yokohama City University Graduate School of Medicine, Yokohama, Japan; ${ }^{14}$ Department of Metabolism and Atherosclerosis, Graduate School of Medicine, Osaka University, Osaka, Japan; ${ }^{15}$ Tokyo Postal Services Agency Hospital, Tokyo, Japan; ${ }^{16}$ Itami City Hospital, Hyogo, Japan; ${ }^{17}$ Division of Endocrinology and Metabolism, Second Department of Internal Medicine, University of the Ryukyus Faculty of Medicine, Okinawa, Japan; ${ }^{18}$ Research Center for Innovative Cancer Therapy, Kurume University, Kurume, Japan; ${ }^{19}$ Department of Community Health and Gerontological Nursing, Faculty of Medicine, Oita University, Oita, Japan; ${ }^{20}$ Division of Endocrinology and Metabolism, Department of Medicine, Kurume University, Kurume, Japan; ${ }^{21}$ Department of Internal Medicine (I), Osaka Medical College, Osaka, Japan; ${ }^{22}$ Division of Endocrinology and Metabolism, Department of Medicine, Nippon Medical School, Tokyo, Japan; ${ }^{23}$ Department of Internal Medicine 1, Faculty of Medicine, Oita University, Oita, Japan and ${ }^{24}$ Department of Medicine and Clinical Science, Kyoto University Graduate School of Medicine, Kyoto, Japan Correspondence: Dr K Hotta, EBM Research Center, Kyoto University Graduate School of Medicine, Yoshida-Konoecho, Sakyo-ku, Kyoto 606-8501, Japan. E-mail: kikukoh@kuhp.kyoto-u.ac.jp

Received 2 November 2011; revised 31 January 2012; accepted 3 February 2012; published online 1 March 2012 
circumference, waist-hip ratio, or visceral fat area (VFA). Waist circumference and waist-hip ratio are commonly used, because they are simple and convenient. However, VFA measured using computed tomography $(\mathrm{CT})$ is one of the most precise methods to assess fat distribution. ${ }^{1,4,5}$ There is an abundance of evidence showing that body fat distribution is influenced by genetic loci. ${ }^{6-8}$ Genome-wide association studies (GWAS) were conducted to identify the loci linked to waist circumference and waist-hip ratio in the Caucasian population. ${ }^{9,10}$ Among the reported loci, we have reported that the rs1558902 and rs1421085 genotypes of the fat mass- and obesityassociated gene (FTO) were significantly associated with VFA, as well as with subcutaneous fat area (SFA) and body mass index (BMI). ${ }^{11}$

GWAS and meta-analysis of GWAS have also identified metabolic syndrome trait-associated genetic variations, including obesity, type 2 diabetes, dyslipidemia and hypertension. ${ }^{12}$ We have previously reported that among the single-nucleotide polymorphisms (SNPs) susceptible to obesity, ${ }^{13-15}$ rs7498665 in the SH2B adaptor protein 1 (SH2B1) gene was associated with VFA, ${ }^{16}$ and that among hypertension-susceptible SNPs, ${ }^{17,18}$ rs1004467 in the cytochrome P450, family 17 , subfamily A, polypeptide 1 (CYP17A1) gene and rs11191548 in the 5'-nucleotidase, cytosolic II (NT5C2) gene were significantly associated with both reduced VFA and SFA in women, indicating a genetic background to central obesity. ${ }^{19}$ Visceral fat obesity is also a risk factor for the development of type 2 diabetes. Several studies have investigated the association of type 2 diabetes loci from GWAS with body fat and BMI. ${ }^{20-22}$ However, there are few reports of the association between type 2 diabetes susceptibility SNPs and visceral fat accumulation. ${ }^{21,23,24}$ Associations between type 2 diabetes susceptibility SNPs, except those exited in the FTO gene and visceral fat mass determined by using more precise method, such as CT and magnetic resonance imaging, have not been elucidated yet. Therefore, we investigated the association between type 2 diabetes susceptibility SNPs and fat distribution (VFA and SFA) as determined by CT.

\section{MATERIALS AND METHODS}

Study subjects

In this study, we enrolled 1279 Japanese subjects from outpatient clinics as described previously. ${ }^{16,19}$ These patients agreed to undergo CT testing (in the supine position) to determine the VFA and SFA values at the umbilical level (L4-L5). Both VFA and SFA values were calculated using the FatScan software program (N2system, Osaka, Japan). ${ }^{25}$ The clinical characteristics of the subjects are summarized in Table 1. Metabolic syndrome and metabolic abnormalities were diagnosed according to the criteria released by the Japanese Committee for the Diagnostic Criteria of Metabolic Syndrome in April 2005., ${ }^{4,5}$ Written informed consent was obtained from each subject, and the protocol was approved by the ethics committee of each institution and of Kyoto University.

\section{DNA extraction and SNP genotyping}

Genomic DNA was extracted using Genomix (Talent Srl, Trieste, Italy) from blood samples collected from each subject. We selected 23 SNPs identified as susceptibility loci for type 2 diabetes by GWAS ${ }^{26-33}$ and constructed Invader probes (Third Wave Technologies, Madison, WI, USA) for the following SNPs: notch 2 (NOTCH2) rs10923931, thyroid adenoma-associated (THADA) rs7578597, peroxisome proliferator-activated receptor $\gamma(P P A R G)$ rs1801282, ADAM metallopeptidase with thrombospondin type 1 motif, 9 (ADAMTS9) rs4607103, insulinlike growth factor 2 mRNA-binding protein 2 (IGF2BP2) rs1470579, vascular endothelial growth factor A (VEGFA) rs9472138, JAZF zinc finger 1 (JAZF1) rs864745, cyclin-dependent kinase inhibitor $2 \mathrm{~A}$ and cyclin-dependent kinase inhibitor 2B (CDKN2A/CDKN2B) rs564398 and rs10811661, hematopoietically expressed homeobox (HHEX) rs1111875 and rs5015480, transcription factor 7-like 2 (TCF7L2) rs7901695, potassium voltage-gated channel, KQT-like subfamily, member 1 (KCNQ1) rs2237892, potassium inwardly-rectifying channel, subfamily J, member 11 (KCNJ11) rs5215 and rs5219, exostosin 2 (EXT2) rs1113132, rs11037909, and rs3740878, melatonin receptor 1B (MTNR1B) rs10830963, dermcidin $(D C D)$ rs1153188, tetraspanin 8/leucine-rich repeat containing G protein-coupled receptor 5 (TSPAN8/LGR5) rs7961581, and FTO rs8050136 and rs9939609. The SNPs were genotyped using Invader assays as previously described. ${ }^{34}$ The success rate of these assays was $>99.0 \%$.

\section{Statistical analysis}

For the additive model, we coded the genotypes as 0,1 or 2 depending on the number of copies of the risk alleles. Risk alleles refer to the type 2 diabetesassociated alleles, according to previous reports. ${ }^{26-33}$ Multiple linear regression analyses were performed to test the independent effects of the risk alleles on BMI, VFA and SFA by taking into account the effects of other variables (i.e., age and gender) that were assumed to be independent of the effect of each SNP. The values of BMI, VFA and SFA were logarithmically transformed before multiple linear regression analysis. Odds ratios and $P$-values adjusted for age, gender and BMI were calculated using multiple logistic regression analysis with genotypes, age, gender and log-transformed BMI as the independent variables. The Hardy-Weinberg equilibrium was assessed using the $\chi^{2}$-test. ${ }^{35}$ All statistical analyses was performed using software R (http://www.r-project.org/). $P$-values were corrected by Bonferroni adjustment and $P<0.0021(0.05 / 23)$ was considered statistically significant.

\section{RESULTS}

The clinical characteristics and genotypes of the subjects are listed in Tables 1 and 2, respectively. All SNPs remained in the HardyWeinberg equilibrium, except rs5215 and rs5219 in KCNJ11 $(P=0.03)$ and rs1153188 in $D C D$ genes $(P=0.04)$. The minor allele frequencies did not diverge from those reported in the HapMap database. The BMI, VFA and SFA values for each SNP genotype are presented in Table 3. Multiple linear regression analyses of the anthropometric parameters with respect to the 23 SNPs analyzed are listed in Table 4. Two SNPs (rs8050136 and rs9939609) in the FTO gene were significantly associated with BMI in an additive model $(P=0.00088$ and 0.0010 , respectively). As the results in Table 3 showed that the dominant model was the best-fit model, we analyzed the above two SNPs in the dominant model. Significant associations of BMI were observed with $\operatorname{rs} 8050136(P=0.00052)$ and rs9939609 $(P=0.00061)$ in a dominant model.

There is no SNP associated with VFA. Only SNPs (rs8050136 and rs9939609) in the FTO gene were marginally associated with VFA $(P<0.05)$. Two SNPs in the FTO gene were significantly associated with SFA ( $P=0.00013$ at $\mathrm{rs} 8050136$ and $P=0.00017$ at $\mathrm{rs} 9939609)$, whereas all other SNPs did not associate with SFA. BMI, VFA and SFA

Table 1 General characteristics of the subjects

\begin{tabular}{lccc}
\hline & Men & Women & Total \\
\hline$n$ & 556 & 723 & 1279 \\
Age (years) & $49.4 \pm 12.2$ & $52.2 \pm 11.3$ & $51.0 \pm 11.8$ \\
BMI $\left(\mathrm{kg} \mathrm{m}^{-2}\right)$ & $30.2 \pm 6.1$ & $28.1 \pm 5.3$ & $29.0 \pm 5.8$ \\
VFA $\left(\mathrm{cm}^{2}\right)$ & $155.3 \pm 67.7$ & $99.8 \pm 53.6$ & $123.9 \pm 66.1$ \\
SFA $\left(\mathrm{cm}^{2}\right)$ & $206.7 \pm 108.6$ & $241.6 \pm 97.2$ & $226.5 \pm 103.7$ \\
Waist circumference $(\mathrm{cm})$ & $97.5 \pm 11.3$ & $91.8 \pm 10.3$ & $94.2 \pm 11.1$
\end{tabular}

$\begin{array}{llll}\text { Prevalence of metabolic disease } & & & \\ \text { Dyslipidemia (\%) } & 293(53) & 244(34) & 537(42) \\ \text { Hypertension (\%) } & 379(68) & 452(63) & 831(65) \\ \text { Impaired fasting glucose (\%) } & 177(32) & 176(24) & 353(28) \\ \text { Metabolic syndrome (\%) } & 248(45) & 162(22) & 410(32)\end{array}$

Abbreviations: BMI, body mass index; SFA, subcutaneous fat area; VFA, visceral fat area. Data are shown as mean \pm s.d. 
Table 2 Type 2 diabetes-susceptible alleles and genotype frequencies in subjects

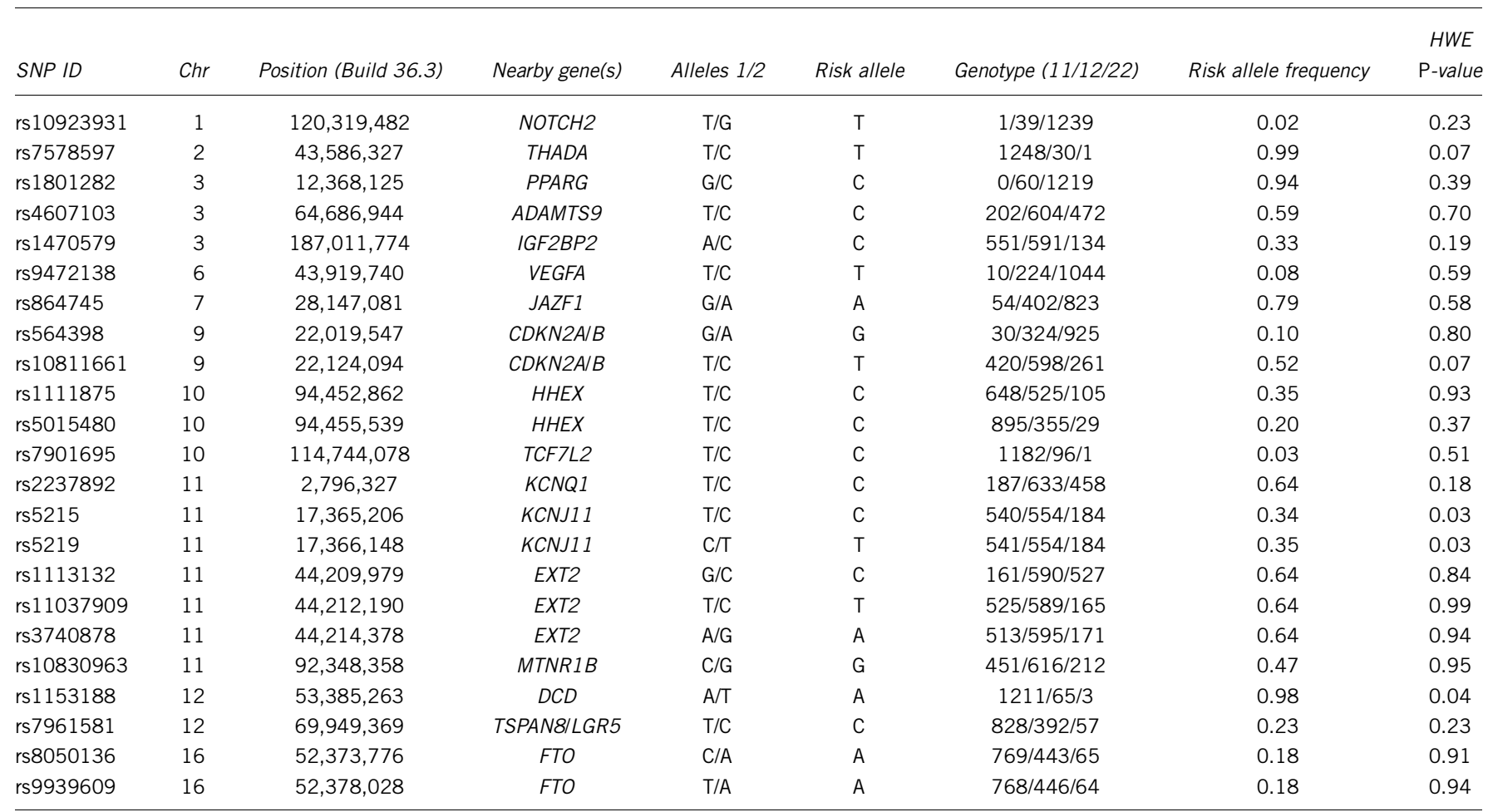

Abbreviations: Chr, chromosome; HWE, Hardy-Weinberg equilibrium; SNP, single-nucleotide polymorphism.

Table 3 Mean BMI, VFA and SFA for 23 type 2 diabetes-susceptible SNPs

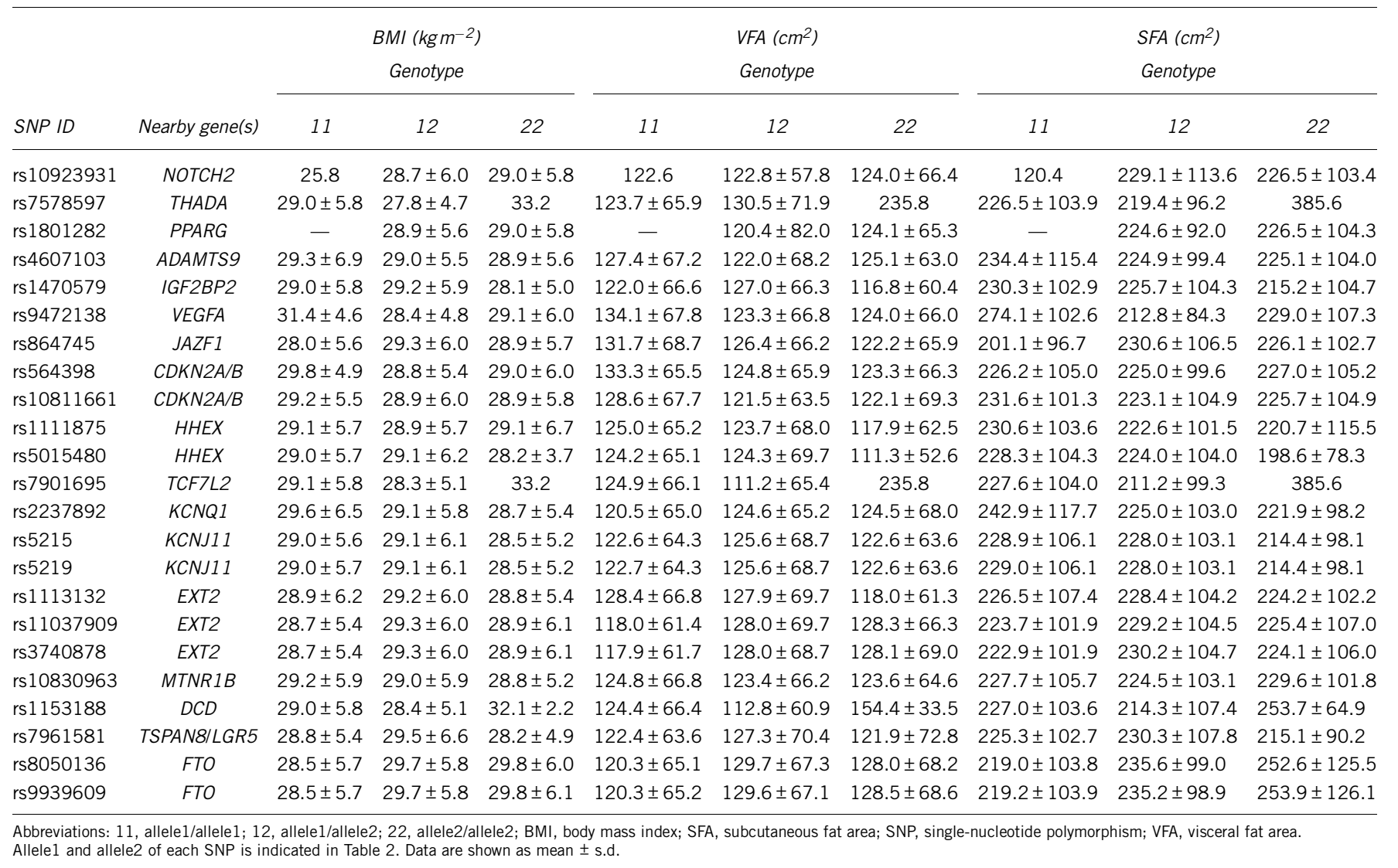


Table 4 Quantitative association results for known type 2 diabetes risk alleles with BMI, VFA, and SFA

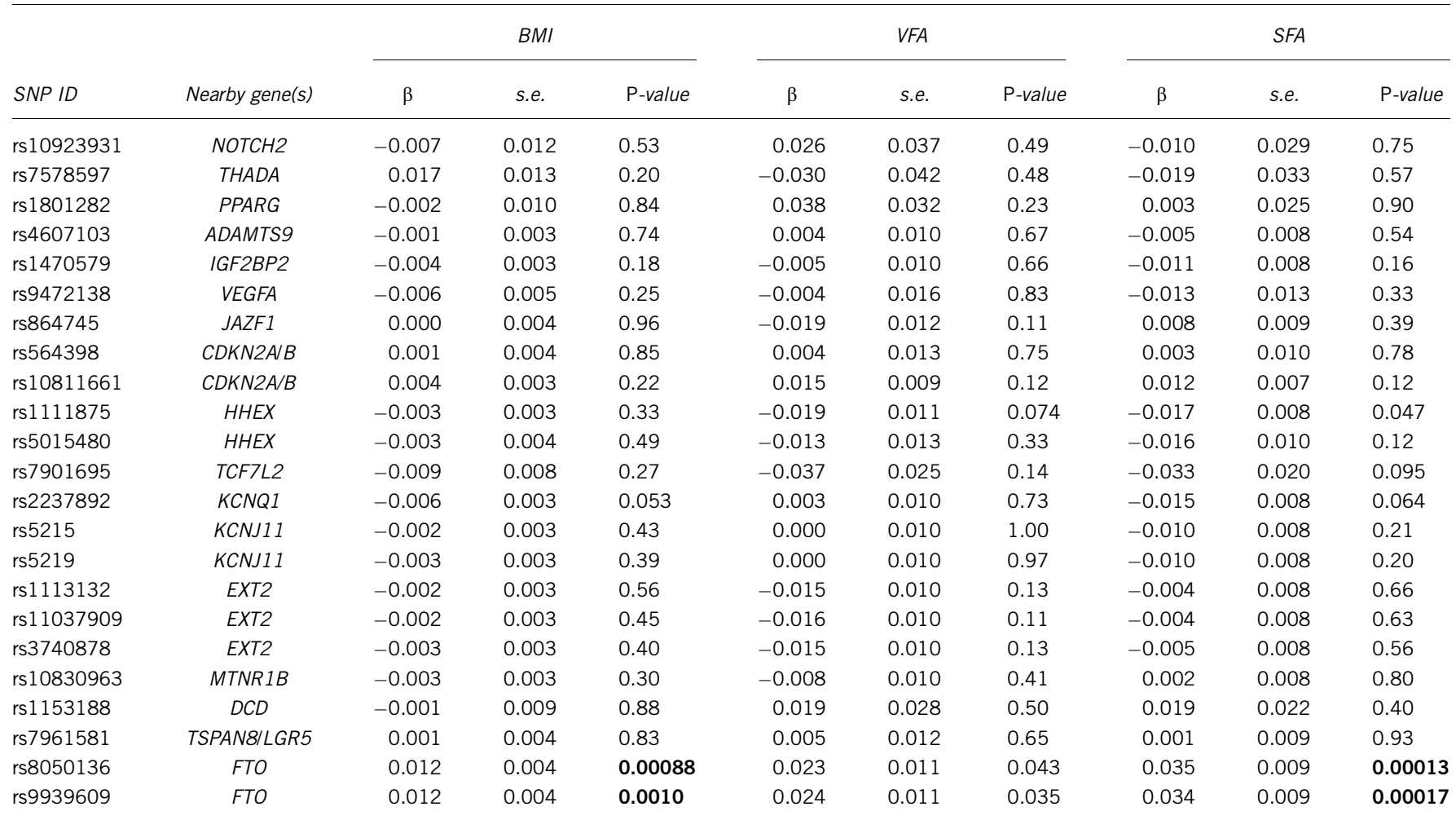

Abbreviations: BMI, body mass index; SFA, subcutaneous fat area; SNP, single-nucleotide polymorphism; VFA, visceral fat area.

Data were derived from a linear regression analysis. BMI, VFA and SFA were adjusted for age and gender, and log-transformed for the analysis. Bold entries indicate $P$-value $<0.0021$.

are known to be affected by gender; therefore, we compared SFA in men and women separately. Associations between FTO rs8050136 and rs9939609 SNPs, and BMI were not significant in either men $(P=0.045$ at $\mathrm{rs} 8050136$ and $P=0.048$ at $\mathrm{rs} 9939609)$ or women $(P=0.0064$ at rs8050136 and $P=0.0075$ at rs9939609) in an additive model. Associations of FTO rs8050136 and rs9939609 SNPs with SFA were not significant in either men $(P=0.0066$ at $\mathrm{rs} 8050136$ and $P=0.0069$ at rs9939609) or women ( $P=0.0058$ at rs8050136 and $P=0.0079$ at rs9939609) in an additive model. This negative association is most likely due to the decrease in the number of each genotype.

We conducted the power analysis of linear regression (additive model) with a significance level of 0.05 , using age and gender as explanatory parameters. The estimated effect sizes per allele (regression coefficients) for logarithmically transformed BMI, VFA and SFA were $0.010,0.017$ and 0.028 , respectively, assuming from the values of rs8050136 and rs9939609 (Table 3). The power of our statistical test was calculated on the basis of these estimated effect sizes and by performing 10000 simulations. When the allele frequency was assumed to be 0.2 , the power was estimated to be 0.71 for BMI, 0.25 for VFA and 0.81 for SFA; however, when the allele frequency was assumed to be 0.1 , the respective powers were estimated to be 0.47 , 0.16 and 0.56 .

Finally, we examined the association of these SNPs with impaired glucose tolerance. Although the number of subjects with impaired glucose tolerance was relatively small $(n=353)$, we could replicate the association of $\mathrm{rs} 10811661$ in the $C D K N 2 A / B$ gene $(P=0.049)$, rs2237892 in the KCNQ1 gene $(P=0.00013)$, and rs1113132 in the EXT2 gene $(P=0.043)$ with impaired glucose tolerance (Table 5).

\section{DISCUSSION}

Obesity, especially visceral fat obesity, is an important risk factor for the development of metabolic syndromes, including type 2 diabetes. Recent GWAS revealed the genetic susceptibility factors for type 2 diabetes. The relationship between type 2 diabetes-associated SNPs and visceral fat obesity is of considerable interest. The CT-based analyses are more accurate for evaluating the association of SNPs with visceral fat mass than BMI, waist circumference, or dual energy X-ray absorptiometry-based abdominal fat mass analysis. ${ }^{4,5}$ Therefore, we investigated the association of type 2 diabetes susceptibility SNPs with VFA and SFA. We demonstrated that only SNPs in the FTO gene are associated with SFA and BMI, but not with VFA. FTO rs8050136 has been shown to be marginally associated with visceral fat mass measured by magnetic resonance imaging in Germany $(P=0.05)^{36}$ and rs9939609 with intra-abdominal adipose tissue estimated by dual energy X-ray absorptiometry in Danish $\left(P=4.7 \times 10^{-3}\right) .{ }^{37}$ SNP rs8050136 has been reported to be marginally associated with VFA measured by CT in African Americans $(P=0.05)$, but not in Hispanic Americans. $^{38}$ Our study showed the weak association VFA and rs8050136 $(P=0.043)$. Although the measurements of visceral fat were different, rs8050136 would be associated with visceral fat accumulation. As the simulation study showed the power of this study for VFA were relatively low (0.16 to 0.35$)$, large sample size would be necessary to confirm the association. SNP rs8050136 has been reported to be marginally associated with SFA measured by CT in African Americans $(P=0.038)$ and in Hispanic Americans $(P=0.019),{ }^{38}$ indicating that $\mathrm{rs} 8050136$ would be related with SFA in various populations. 
Table 5 Association of type 2 diabetes-associated loci alleles and impaired fasting plasma glucose

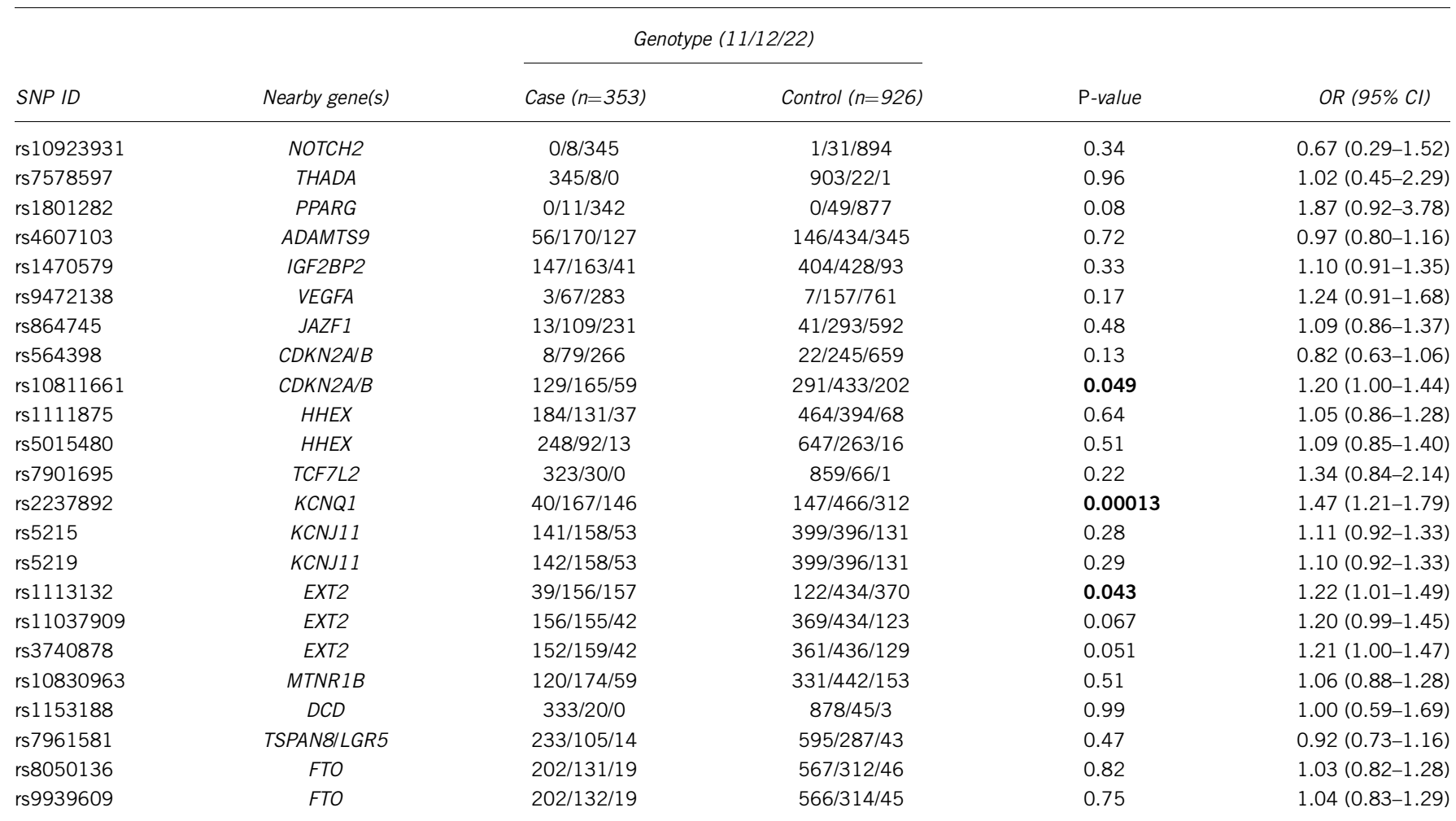

Abbreviation: BMI, body mass index; $\mathrm{Cl}$, confidence interval; OR, odds ratio; SNP, single-nucleotide polymorphism.

Data were derived from logistic regression analysis. $P$-value and ORs were adjusted for age, gender and BMI. BMI was log-transformed for the analysis. Bold entries indicate $P$-value $<0.05$.

Other type 2 diabetes susceptibility SNPs were not associated with BMI, VFA or SFA. Pecioska et al. ${ }^{21}$ reported that among the type 2 diabetes loci (SLC30A8, CDKN2A/B, CDKAL1, IGF2BP2, HHEX, PPARG, KCNJ11, TCFL2 and FTO), only FTO rs8050136 was associated with BMI, fat mass index (fat mass/height/height) and waist circumference. Our results were in agreement with those reported by Pecioska et al. ${ }^{21}$ The $\mathrm{T}$ allele of rs2237892 in the KCNQ1 gene was reported to be associated with increased waist circumference $(P=0.043) .{ }^{24}$ However, the subjects with $\mathrm{T}$ allele had lower VFA in our study. A lack of association of type 2 diabetes loci, and VFA and SFA may be due to the low power of this study. The associations between SNPs and type 2 diabetes were adjusted for BMI, age and gender. Thus, SNPs associated with both obesity and type 2 diabetes would be excluded in the screening stage. Indeed, FTO was first discovered as a susceptibility gene for type 2 diabetes in an analysis that was not adjusted for BMI; after adjustment for BMI, the effect on type 2 diabetes was abolished. ${ }^{39}$

Most risk factors for type 2 diabetes are believed to act through perturbation of insulin secretion rather than through insulin action (insulin resistance) ${ }^{40}$ It is believed that $C D K N 2 A$ and $C D K N 2 B$ genes are involved in reducing $\beta$-cell mass, whereas MTNR1B, TCFL2 and KCNJ11 genes may be involved in $\beta$-cell dysfunction. Only the FTO gene can act through insulin resistance caused by obesity. Our results, demonstrating no significant association of type 2 diabetes susceptibility SNPs with VFA or SFA, except for SNPs in the FTO gene, confirm the above mechanism for the development of type 2 diabetes.

In summary, we showed that two SNPs (rs8050136 and rs9939609) in the FTO gene are significantly associated with SFA and BMI, and that other SNPs susceptible for type 2 diabetes are not associated with fat distribution. Our results suggest that only SNPs in the FTO gene are involved in the mechanism of insulin resistance.

\section{CONFLICT OF INTEREST}

The authors declare no conflict of interest.

\section{ACKNOWLEDGEMENTS}

This study was supported by a Grant-in-Aid from the Ministry of Education, Science, Sports and Culture of Japan (21591186 to K Hotta, 23791027 to $\mathrm{AK}$, and 23701082 to $\mathrm{TK})$.

1 Carr, D. B., Utzschneider, K. M., Hull, R. L., Kodama, K., Retzlaff, B. M., Brunzell, J. D. et al. Intra-abdominal fat is a major determinant of the National Cholesterol Education Program Adult Treatment Panel III criteria for the metabolic syndrome. Diabetes 53, 2087-2094 (2004).

2 Matsuzawa, Y. Therapy insight: adipocytokines in metabolic syndrome and related cardiovascular disease. Nat. Clin. Pract. Cardiovasc. Med. 3, 35-42 (2006).

3 Hotta, K., Funahashi, T., Bodkin, N. L., Ortmeyer, H. K., Arita, Y., Hansen, B. C. et al. Circulating concentrations of the adipocyte protein adiponectin are decreased in parallel with reduced insulin sensitivity during the progression to type 2 diabetes in rhesus monkeys. Diabetes 50, 1126-1133 (2001).

4 Mastuzawa, Y. Metabolic syndrome-definition and diagnostic criteria in Japan. J. Atheroscler. Thromb. 12, 301 (2005).

5 Arai, H., Yamamoto, A., Matsuzawa, Y., Saito, Y., Yamada, N., Oikawa, S. et al. Prevalence of metabolic syndrome in the general Japanese population in 2000. J. Atheroscler. Thromb. 13, 202-208 (2006).

6 Selby, J. V., Newman, B., Quesenberry, C. P. Jr, Fabsitz, R. R., Carmelli, D., Meaney, F. J. et al. Genetic and behavioral influences on body fat distribution. Int. J. Obes. 14, 593-602 (1990).

7 Rose, K. M., Newman, B., Mayer-Davis, E. J. \& Selby, J. V. Genetic and behavioral determinants of waist-hip ratio and waist circumference in women twins. Obes. Res. 6 , 383-392 (1998). 
8 Souren, N. Y., Paulussen, A. D., Loos, R. J., Gielen, M., Beunen, G., Fagard, R. et al. Anthropometry, carbohydrate and lipid metabolism in the East Flanders Prospective Twin Survey: heritabilities. Diabetologia 50, 2107-2116 (2007)

9 Lindgren, C. M., Heid, I. M., Randall, J. C., Lamina, C., Steinthorsdottir, V., Qi, L. et al. Genome-wide association scan meta-analysis identifies three loci influencing adiposity and fat distribution. PLoS Genet. 5, e1000508 (2009).

10 Heard-Costa, N. L., Zillikens, M. C., Monda, K. L., Johansson, Å., Harris, T. B., Fu, M. et al. NRXN3 is a novel locus for waist circumference: a genome-wide association study from the CHARGE Consortium. PLoS Genet. 5, e1000539 (2009).

11 Hotta, K., Nakamura, M., Nakamura, T., Matsuo, T., Nakata, Y., Kamohara, S. et al. Polymorphisms in NRXN3, TFAP2B, MSRA, LYPLAL1, FTO and MC4R and their effect on visceral fat area in the Japanese population. J. Hum. Genet. 55, 738-742 (2010).

12 Hindorff, L. A., Sethupathy, P., Junkins, H. A., Ramos, E. M., Mehta, J. P., Collins, F. S. et al. Potential etiologic and functional implications of genome-wide association loci for human diseases and traits. Proc. Natl Acad. Sci. USA 106, 9362-9367 (2009).

13 Thorleifsson, G., Walters, G. B., Gudbjartsson, D. F., Steinthorsdottir, V., Sulem, P. Helgadottir, A. et al. Genome-wide association yields new sequence variants at seven loci that associate with measures of obesity. Nat. Genet. 41, 18-24 (2009).

14 Willer, C. J., Speliotes, E. K., Loos, R. J., Li, S., Lindgren, C. M., Heid, I. M. et al. Six new loci associated with body mass index highlight a neuronal influence on body weight regulation. Nat. Genet. 41, 25-34 (2009).

15 Meyre, D., Delplanque, J., Chèvre, J. C., Lecoeur, C., Lobbens, S., Gallina, S. et al. Genome-wide association study for early-onset and morbid adult obesity identifies three new risk loci in European populations. Nat. Genet. 41, 157-159 (2009).

16 Hotta, K., Kitamoto, T., Kitamoto, A., Mizusawa, S., Matsuo, T., Nakata, Y. et al. Computed tomography analysis of the association between SH2B1 rs7498665 singlenucleotide polymorphism and visceral fat area. J. Hum. Genet. 56, 716-719 (2011).

17 Levy, D., Ehret, G. B., Rice, K., Verwoert, G. C., Launer, L. J., Dehghan, A. et al. Genome-wide association study of blood pressure and hypertension. Nat. Genet. 41, 677-687 (2009).

18 Newton-Cheh, C., Johnson, T., Gateva, V., Tobin, M. D., Bochud, M., Coin, L. et al. Genome-wide association study identifies eight loci associated with blood pressure. Nat. Genet. 41, 666-676 (2009).

19 Hotta, K., Kitamoto, A., Kitamoto, T., Mizusawa, S., Teranishi, H., Matsuo, T. et al. Genetic variations in the CYP17A1 and NT5C2 genes are associated with a reduction in visceral and subcutaneous fat areas in Japanese women. J. Hum. Genet. 57, 46-51 (2012).

20 Rong, R., Hanson, R. L., Ortiz, D., Wiedrich, C., Kobes, S., Knowler, W. C. et al. Association analysis of variation in/near FTO, CDKAL1, SLC3OA8, HHEX, EXT2, IGF2BP2, LOC387761, and CDKN2B with type 2 diabetes and related quantitative traits in Pima Indians. Diabetes 58, 478-488 (2009).

21 Pecioska, S., Zillikens, M. C., Henneman, P., Snijders, P. J., Oostra, B. A., van Duijn, C. M. et al. Association between type 2 diabetes loci and measures of fatness. PLoS One 5 , e8541 (2010).

22 Ng, M. C., Park, K. S., Oh, B., Tam, C. H., Cho, Y. M., Shin, H. D. et al. Implication of genetic variants near TCF7L2, SLC30A8, HHEX, CDKAL1, CDKN2A/B, IGF2BP2, and FTO in type 2 diabetes and obesity in 6,719 Asians. Diabetes 57, 2226-2233 (2008).

23 Thorsby, P. M., Midthjell, K., Gjerlaugsen, N., Holmen, J., Hanssen, K. F., Birkeland, K. I. et al. Comparison of genetic risk in three candidate genes (TCF7L2, PPARG, KCNJ11) with traditional risk factors for type 2 diabetes in a population-based study - the HUNT study. Scand. J. Clin. Lab. Invest. 69, 282-287 (2009).
24 Liu, Y., Zhou, D. Z., Zhang, D., Chen, Z., Zhao, T., Zhang, Z. et al. Variants in KCNQ1 are associated with susceptibility to type 2 diabetes in the population of mainland China. Diabetologia 52, 1315-1321 (2009).

25 Yoshizumi, T., Nakamura, T., Yamane, M., Islam, A. H., Menju, M., Yamasaki, K. et al. Abdominal fat: standardized technique for measurement at CT. Radiology 211, 283-286 (1999).

26 Grant, S. F., Thorleifsson, G., Reynisdottir, I., Benediktsson, R., Manolescu, A., Sainz, J. et al. Variant of transcription factor 7-like 2 (TCF7L2) gene confers risk of type 2 diabetes. Nat. Genet. 38, 320-323 (2006).

27 Wellcome Trust Case Control Consortium. Genome-wide association study of 14,000 cases of seven common diseases and 3,000 shared controls. Nature 447, 661-678 (2007).

28 Sladek, R., Rocheleau, G., Rung, J., Dina, C., Shen, L., Serre, D. et al. A genome-wide association study identifies novel risk loci for type 2 diabetes. Nature 445, 881-885 (2007).

29 Diabetes Genetics Initiative of Broad Institute of Harvard and MIT, Lund University and Novartis Institutes of BioMedical Research, Saxena, R., Voight, B. F., Lyssenko, V., Burtt, N. P., de Bakker, P. I., Chen, H. et al. Genome-wide association analysis identifies loci for type 2 diabetes and triglyceride levels. Science 316, 1331-1336 (2007).

30 Zeggini, E., Scott, L. J., Saxena, R., Voight, B. F., Marchini, J. L., Hu, T. et al. Metaanalysis of genome-wide association data and large-scale replication identifies additional susceptibility loci for type 2 diabetes. Nat. Genet. 40, 638-645 (2008).

31 Unoki, H., Takahashi, A., Kawaguchi, T., Hara, K., Horikoshi, M., Andersen, G. et al. SNPs in KCNQ1 are associated with susceptibility to type 2 diabetes in East Asian and European populations. Nat. Genet. 40, 1098-1102 (2008).

32 Yasuda, K., Miyake, K., Horikawa, Y., Hara, K., Osawa, H., Furuta, H. et al. Variants in KCNQ1 are associated with susceptibility to type 2 diabetes mellitus. Nat. Genet. 40, 1092-1097 (2008).

33 Lyssenko, V., Nagorny, C. L., Erdos, M. R., Wierup, N., Jonsson, A., Spégel, P. et al. Common variant in MTNR1B associated with increased risk of type 2 diabetes and impaired early insulin secretion. Nat. Genet. 41, 82-88 (2009).

34 Ohnishi, Y., Tanaka, T., Ozaki, K., Yamada, R., Suzuki, H. \& Nakamura, Y. A highthroughput SNP typing system for genome-wide association studies. J. Hum. Genet. 46, 471-477 (2001)

35 Nielsen, D. M., Ehm, M. G. \& Weir, B. S. Detecting marker-disease association by testing for Hardy-Weinberg disequilibrium at a marker locus. Am. J. Hum. Genet. 63, 1531-1540 (1998).

36 Haupt, A., Thamer, C., Machann, J., Kirchhoff, K., Stefan, N., Tschritter, O. et al. Impact of variation in the FTO gene on whole body fat distribution, ectopic fat, and weight loss. Obesity 16, 1969-1972 (2008).

37 Kring, S. I., Holst, C., Zimmermann, E., Jess, T., Berentzen, T., Toubro, S. et al. FTO gene associated fatness in relation to body fat distribution and metabolic traits throughout a broad range of fatness. PLoS One 3, e2958 (2008).

38 Wing, M. R., Ziegler, J., Langefeld, C. D., Ng, M. C., Haffner, S. M., Norris, J. M. et al. Analysis of FTO gene variants with measures of obesity and glucose homeostasis in the IRAS Family Study. Hum. Genet. 125, 615-626 (2009).

39 Frayling, T. M., Timpson, N. J., Weedon, M. N., Zeggini, E., Freathy, R. M., Lindgren, C. M. et al. A common variant in the FTO gene is associated with body mass index and predisposes to childhood and adult obesity. Science 316, 889-894 (2007).

40 McCarthy, M. I. Genomics, type 2 diabetes, and obesity. N. Engl. J. Med. 363, 2339-2350 (2010). 